\title{
Solitary and bilateral choroidal metastasis revealing breast cancer
}

\begin{abstract}
Introduction: Breast cancer is complicated by intraocular metastases in $8-10 \%$ of cases and these metastases occur most often in patients already known with cancer. Revealing forms are exceptional as well as bilateral involvement. We report an original case characterized by bilateral and solitary (without other visceral or bone secondary locations) choroidal metastasis revealing breastneoplasia.
\end{abstract}

Observation: 38 years old patient with non otable medical history presented to a progressive decrease in visual acuity of the right eye lasting for two weeks and suddenly worsened in the last two days. Ophthalmologic examination showed a larged etachment of the right retina with retinal infiltrates in both sides. The oculoorbital ultrasound exam showed the presence of bilateral choroidal echogenic, well circumscribed, and homogeneous tissular formations. The exploration was completed by oculo-orbital MRI confirming the same findings. Research of the primitive objectified left breast carcinoma. Reviewing other possible metastases was negative. The patient was assigned to radiotherapy but the evolution was unfavorable with worsening of visual impairment and persistence of choroidal metastases on ultrasound control after one month.

Conclusion: An ophthalmologic evaluation with fundus exam may be indicated in the staging of breast neoplasm. In other hand, breast examination should be systematic to the discovery of achoroïdal secondary tumor.
Volume 8 Issue 3 - 2018

\author{
Mayeda Ben Hamed, Nesrine Regaïeg, \\ Nesrine Belgacem, Hassen Baili, Najla \\ Lassoued, Salem Bouomrani \\ Department of Internal medicine, Military Hospital of Gabes, \\ Tunisia
}

Correspondence: Salem Bouomrani, Department of Internal medicine, Military Hospital of Gabes, Tunisia, Tel +0021698977555, Email salembouomrani@yahoo.fr

Received: May II, 2018 | Published: May 22, 2018

Keywords: cancer, breast, metastasis, choroïd

\section{Introduction}

Breast cancer is complicated by intra-ocular metastases in $8-10 \%$ of cases and these metastases occur in $77-90 \%$ of already-known cancer patients. ${ }^{1,2}$ The revealing forms remain exceptional ${ }^{3}$ as well as bilateral involvement. ${ }^{2}$

We report a particular observation characterized by bilateral and isolated choroidal metastases (without other visceral or bone secondary sites) indicative of mammary neoplasia.

\section{Case report}

A 38-year-old patient, with no notable pathological history, presented for a progressive decrease in visual acuity of the right eye that had been evolving for two weeks and had been brutally aggravated in the last two days. Ophthalmological examination with fundus showed serous and extensive detachment of the right retina with sub-retinal infiltrates on both sides. B-mode oculo-orbital ultrasonography with a high-frequency probe showed the presence of well-defined echogenic formations of homogeneous choroidal and bilateral tissue echostructure (Figure 1-3) suggestive of metastases. The exploration was completed by oculo-orbital magnetic resonance imaging confirming the same findings. The search for the primitive objectified a left breast carcinoma. She had a hard, irregular nodule two centimeters from the supero-outer quadrant of the left breast. Echo-mammography showed stellar nodular opacity at the left supero-external quadrant measuring two centimeters and containing micro-calcifications. This opacity was rated ACR 4 (American College of Radiology) and highly suspect.
The CA15-3 level was elevated to $85 \mathrm{IU} / \mathrm{ml}$ (normal $<35 \mathrm{IU} / \mathrm{ml}$ ) and the nodule cyto-puncture found infiltrating ductal adenocarcinoma with trabecular carcinomatous cells, pleomorphic nuclei, marked atypia, and many mitoses; grade III SBR (histo-prognosis grade of Scarff Bloom and Richardson). There were no associated axillary lymphadenopathies, neither homo nor contralateral. Extension assessment for other metastases was negative (abdominal ultrasound, standard chest X-ray, thoraco abdominal CT scan, brain MRI and bone scintigraphy). The patient was given immediate radiotherapy for secondary ocular lesions given the visual risk. After four courses of radiotherapy, the evolution was unfavorable with worsening of the visual deficit and persistence of choroidal metastases unchanged on the control ultrasound done a month later. Surgery for breast cancer was refused by the patient.

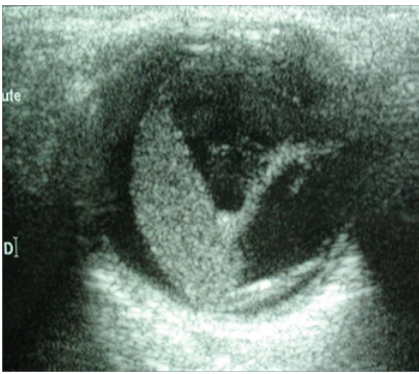

1

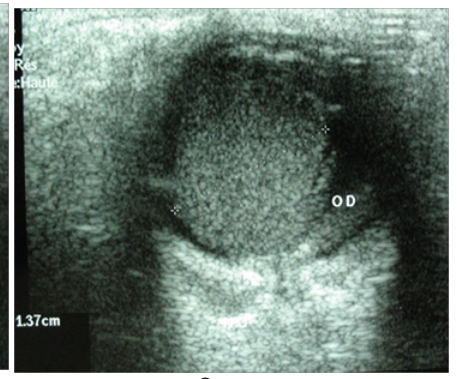

2
Figure I \&2 Ocular B ultrasonography in transocular sections: right choroidal tumor in lateral (I) and front (2) view with a diameter of $1.37 \mathrm{~cm}$. 


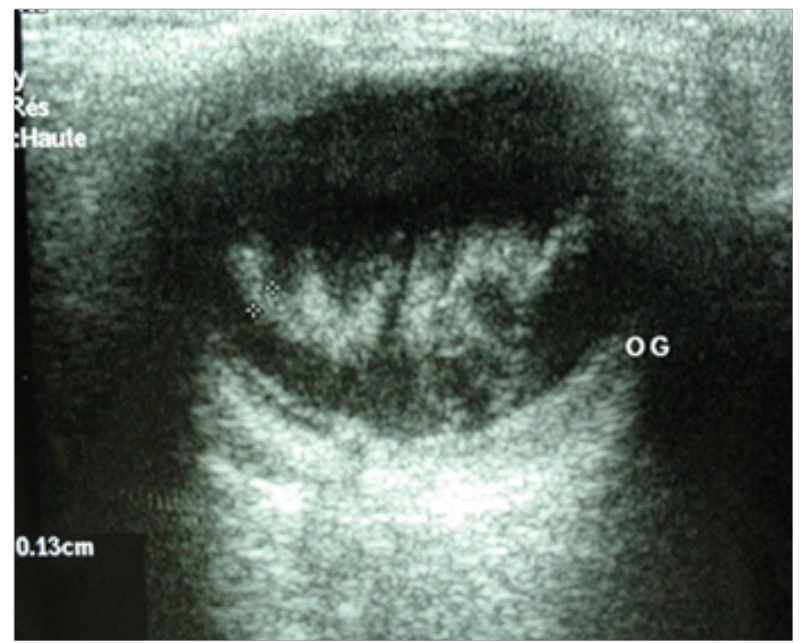

Figure 3 Ocular B ultrasonography in transocular section: left choroidal tumor in lateral view.

\section{Discussion}

Metastases are the most common intraocular tumors in adults ${ }^{4}$ and the choroid is the typical site of these secondary tumors. ${ }^{4-6}$ They are indicative of solid cancer in 15 to $30 \%$ of cases. ${ }^{5}$ The primitives most frequently found are pulmonary, digestive, pancreatic, renal, breast cancer for women and prostatic for men. ${ }^{4,5,7}$

Indeed, $85 \%$ of all intraocular metastases of breast cancer are located in the choroid. ${ }^{6}$ Although they are considered "classic" in breast cancer, ocular secondary sites complicate only 8 to $10 \%$ of these cancers ${ }^{1,2}$ This frequency, however, remains very underestimated since systematic research found microscopic uvei metastases in 37 to $41 \%$ of subjects with breast cancer. ${ }^{2}$ This major recrudescence is explained by the hyper-vascularized nature of the uveal ocular body. ${ }^{2}$

Choroidal metastases indicative of breast cancer remain exceptional ${ }^{4-6}$ since these secondary locations mainly complicate previously known mammary neoplasias ${ }^{3,6}$ indeed in the large series of Demirci H et al. ${ }^{6}$ grouping 264 patients with uveeal metastases related to breast cancer, the attack was indicative of primary neoplasia in only seven patients, or $3 \%$ of. ${ }^{6}$ On the other hand, the choroid represented the first systemic metastatic site in $16 \%$ of previously known subjects with breast cancer. ${ }^{6}$

The secondary choroidal localization of breast cancer can be associated with other intraocular metastatic sites (uveal, optic nerve, sclera, retina.), ${ }^{2}$ conceal central nervous system metastasis ${ }^{6,8}$ or to announce a tumor recurrence. ${ }^{9}$

Solitary forms (without other systemic metastatic locations) are exceptional ${ }^{10}$ and bilateral forms are rare ${ }^{4,5}$ but it should be noted that in more than half of the cases, contralateral eye involvement remains infra-clinical. and would be discovered only by systematic ocular examination. ${ }^{6}$ Choroidal metastases are also reported with human breast cancer. ${ }^{11}$

Clinically, these secondary choroidal tumors are manifested by visual blur, photophobia, visual acuity, ocular pain, eye redness and visual field impairment $t^{6,7}$ and remain totally asymptomatic in $7 \%$ of cases. ${ }^{6,12}$ The therapeutic management of these metastases is purely palliative to preserve as much as possible a satisfactory visual acuity; it is mainly based on external radiotherapy and anti-cancer poly-chemotherapy adapted to the type of primary neoplasia., ${ }^{4,6,13}$ Radiotherapy provides a satisfactory response in 75 to $85 \%$ of cases. ${ }^{6,13}$

Surgery has no place in this treatment and even the few attempts at tumor reduction in large metastases are associated with increased morbidity and mortality. ${ }^{4}$

\section{Conclusion}

An ophthalmological examination with a fundus eye is indicated in the extension assessment of a breast cancer even in the absence of ocular symptomatology; similarly breast examination should be systematic in the presence of a single or bilateral choroidal tumor.

\section{Acknowledgments}

None.

\section{Conflicts of interest}

The author declares there is no conflict of interest.

\section{References}

1. Niu YJ, Liu FL, Zhou ZY, et al. Clinical and pathological analysis of choroidal metastatic carcinoma. Zhonghua Yan Ke Za Zhi. 2006;42(7):5804.

2. Biswas J, Ho TC, Bhavsar K. Bilateral metastasis to the retina, choroids and optic nerve from breast cancer: a clinicopathological case. Indian $J$ Ophthalmol. 2007;55(1):71-2.

3. Wickremasinghe $\mathrm{S}$, Dansingani KK, Tranos $\mathrm{P}$, et al. Ocular presentations of breast cancer. Acta Ophthalmol Scand. 2007;85(2):133-42.

4. Lam M, Lee J, Teoh S, et al. Choroidal metastasis as the presenting feature of a non-small cell lung carcinoma with no apparent primary lesion identified by X-ray: A case report. Oncol Lett. 2014;8(4):1886-1888.

5. Pugnet G, Arista S, Martin-Blondel G, et al. Métastase choroïdienne révélant un cancer du sein. Rev Med Interne. 2007;28(11):790-2.

6. Demirci H, Shields CL, Chao AN, et al. Uveal metastasis from breast cancer in 264patients. Am J Ophthalmol. 2003;136(2):264-71.

7. Zhang HR, Ma ZZ, Feng Y, Guo T. Clinical characteristics of choroidalmetastasis. Zhonghua Yan Ke Za Zhi.2009;45(4):301-8.

8. Sirimaharaj M, Hunyor AP, Chan WC, et al. Unsual ocular metastasis from breast cancer. Clin Experiment Ophthalmol. 2006;34(1):74-6.

9. Sánchez R, Betancourt L, Sánchez A, et al. Choroidal metastasis as first manifestation of systemic recurrence of breast cancer. Breast $J$. 2008;14(5):498-500.

10. Rundle P, Rennie I. Photodynamic therapy for solitary retinalmetastasis from breast carcinoma. Eye (Lond). 2006;20(12):1410-2.

11. Shome D, Jayadev C, Gadgil D, et al. Systemic chemotherapy and tamoxifen induced regression of choroidal metastasis from a breast carcinoma in a male. Indian J Ophthalmol. 2007;55:475-7.

12. Wiegel T, Kreusel KM, Bornfeld N, et al. Frequency of asymptomatic choroidal metastasis in patients with disseminated breast cancer: results of a prospective screening programme. Br J Ophthalmol. 1998;82(10):115961.

13. Amichetti M, Caffo O, Minatel E, et al. Ocular metastases from breast carcinoma: A multicentric retrospective study. Oncol Rep. 2000;7(4):7615. 